\title{
HIPPARCOS ASTROMETRIC RESULTS
}

\author{
J. KOVALEVSKY \\ OCA /CERGA \\ UMR CNRS 6527 \\ Av. Copernic, 06130-Grasse (France)
}

\begin{abstract}
The astrometric results of Hipparcos include the positions at epoch (1991.25), the proper motion in the new IAU extragalactic reference system (ICRS), and parallaxes for about 118000 stars. One dimensional positions are also given for 48 asteroids and 3 satellites. Due to the non-isotropy of the scanning law, the uncertainties are position dependent. Some indications of the remaining correlations are given. Various tests and comparisons show that systematic errors in parallax, if any, are not larger than 0.1 millisecond of arc.
\end{abstract}

\section{DESCRIPTION OF THE CATALOGUE}

The Hipparcos catalogue, now published as ESA publication SP 1200 (July-September 1997) and in electronic form, in particular from the CDS, provides astrometric data for 117955 stars almost uniformly distributed over the sky. It includes a survey of about 52000 bright stars complete to a limiting visual magnitude $V$ such that, if $b$ is the galactic latitude :

$V \leq 7.9+1.1 \sin |b|$ for spectral types earlier or equal to G5

$V \leq 7.3+1.1 \sin |b|$ for spectral types later than G5

The other 66000 stars were selected for their astrophysical or astrometric interest or in order to ensure a minimum density in star poor regions.

\section{THE ASTROMETRIC PROPERTIES OF THE CATALOGUE}

They can be summarized by the few following features.

- The positions and the proper motions are referred to the ICRS (International Celestial Reference System; Ma and Feissel, 1997) which is fixed with respect to distant extragalactic radio-sources. This link has been realised from a discussion of eleven different determinations using radio-interferometry, photographic plates, star catalogues linked to galaxies, HST observations, and analysis of Earth rotation determined by Hipparcos stars. The method used and the results obtained by each team are described in Volume 3 of the catalogue (ESA 1997) and with many additional details in Kovalevsky et al (1997). The remaining offset at epoch (J1991.25) with ICRS axes has an uncertainty at one sigma level of 0.6 mas. The uncertainty in stopping the spin of the catalogue axes is $0.25 \mathrm{mas} / \mathrm{yr}(1 \mathrm{sigma})$. This means that in using the catalogue for galactic dynamics, one has to enter this latter uncertainty in the error budget.

- The median precision of positions at epoch for stars with Hipparcos magnitudes $H_{p}<9$ mag is 0.77 mas in RA and 0.64 mas in declination.

- The median precision of proper motions for the same selection of stars is 0.88 mas/yr in RA and $0.74 \mathrm{mas} / \mathrm{yr}$ in declination.

- The median precision of parallaxes for the same selection of stars is 0.97 mas. About 21000 stars have a relative uncertainty in parallaxes smaller than $10 \%$, out of which, 3000 are double or multiple systems. 
- There are 23882 solved or suspected double stars. The astrometric parametre uncertainties are generally somewhat poorer than for single stars.

- In addition, results of the observations of 48 asteroids and 3 planetary satellites (JII - Europa, SVI Titan and SVIII Iapetus) are given as a one dimensional quantity: the projection of the position of the object on a reference great circle for each observed transit. The observations as reduced by FAST and NDAC are both given without merging, because the reduction procedures are not identical. The standard uncertainties of each data point range between 5 and 30 mas, depending upon the magnitude of the object. In total, 2887 NDAC and 2657 FAST asteroid positions are provided. Almost all FAST positions are in addition complemented by a determination of the magnitude. Practically only NDAC provided the 107 positions of the planetary satellites.

\section{PROPERTIES OF THE UNCERTAINTIES}

The scanning law of the satellite was not isotropic: all the scans had to be inclined by at least $43^{\circ}$ to the ecliptic in order to minimize the solar strayglight. The consequences are that parametres in declination are better determined than in right ascension. In addition, in the mean, the standard errors are larger by a factor of about 1.6 in proper motions in RA and in parallaxes for stars around the ecliptic than around the ecliptic poles. This factor is smaller than 1.1 for proper motions in declination. At intermediate latitudes, the situation is of course somewhere in-between (see figure 1).
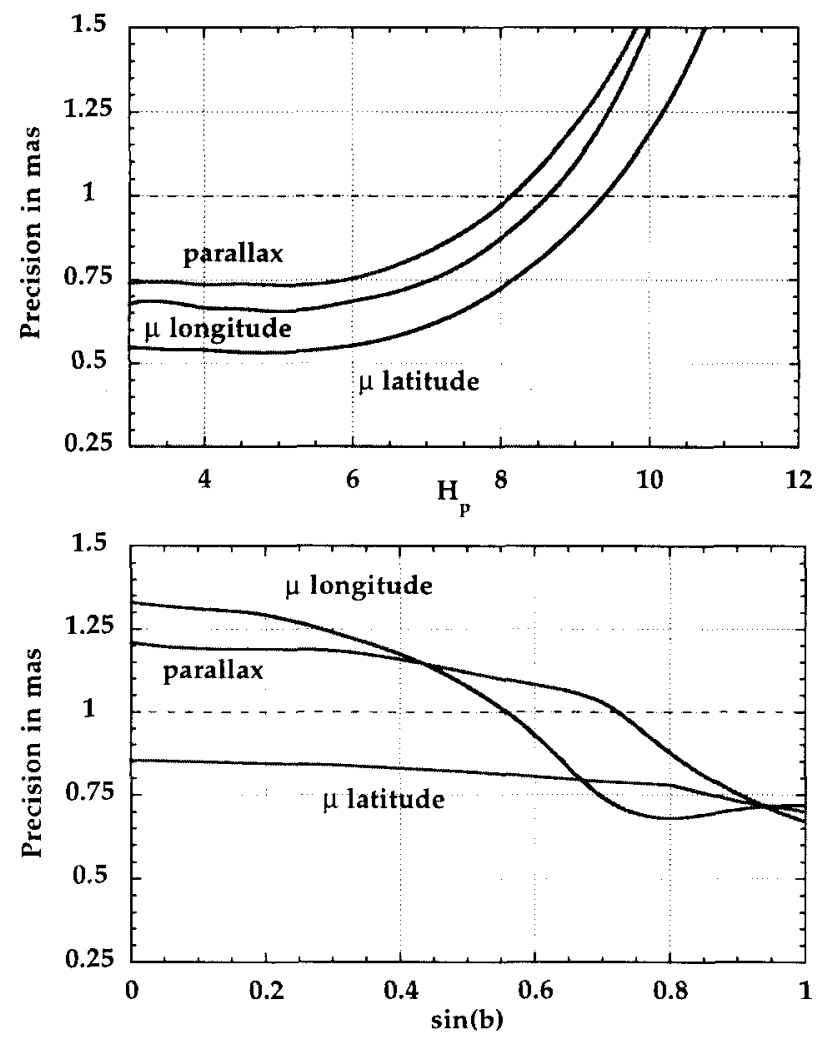

Figure 1. Mean precision of parallaxes and proper motion for single stars as functions of Hipparcos magnitudes (above) and the ecliptic latitude (below). 
The uncertainties also increase with magnitude. They are approximately constant up to magnitude $H_{p}=6.5$ for which $\sigma_{\pi} \cong 0.75 \mathrm{mas} ; \sigma_{\alpha \cos \delta}=0.65 \mathrm{mas} / \mathrm{yr}$ and $\delta_{\mu \delta}=0.55 \mathrm{mas} / \mathrm{yr}$. Then they steadily increase to the double of these values for $H_{p}=10$.

Because of the scanning symmetry about the plane of the ecliptic, the correlations are small when expressed in ecliptic coordinates between $l$ and $b$ and between the components of proper motions $(<0.2)$ but reach 0.4 when one uses equatorial coordinates. This is an artefact, the increase results only from breacking down the symmetry. Correlations between $\alpha$ and $\mu_{\alpha} \cos \delta$ or $\delta$ and $\mu_{\delta}$ may be larger than 0.5 , but again is an artefact whenever the actual mean observation time is far from the adopted J1991.25 epoch. It can be reduced to a minimum by referring stars to their mean observation time.

The only systematic effect concerns the correlation between the right ascension and the parallax. It reaches -0.4 around $\mathrm{RA}=6 \mathrm{~h}$ and +0.4 around $\mathrm{RA}=18 \mathrm{~h}$ for latitudes between $\pm 60^{\circ}$. This effect is well understood as a consequence of an uneven distribution of observations in time due to payload failures, provoking an unbalanced distribution of longitude differences with the Sun.

\section{SYSTEMATIC ERRORS}

Several methods were used to test whether Hipparcos catalogue has significant biases, particularly in the parallaxes.

- A comparison of Hipparcos with the ground based 88 optical parallaxes determined by the USNO 61-inch reflector gave a median difference of $+0.2 \pm 0.35$ mas and parallaxes determined by VLBI showed similar identity.

- The average parallax of 38 non double stars in the Magellanic Clouds was found to be -0.1 \pm 0.23 mas.

- Comparing between distances of moving open clusters as determined from classical ground based astronomy and the distance of some of their members provided by Hipparcos yielded a mean difference of $0.04 \pm 0.06$ mas.

- Cluster distances determined by multiband photometry were compared with Hipparcos parallaxes. One has obtained $-0.05 \pm 0.05$ mas.

These results all converge in estimating that if there is zero-point error in Hipparcos parallaxes, it is certainly below 0.1 mas.

A similar analysis cannot be made for proper motions, mainly because of regional errors present in all ground-based catalogues. However a comparison with FK5 proper motions, permitted to point out one difficulty: the Hipparcos proper motions are quasi-instantaneous proper motions, in contrast with FK5, based upon 50 or more years of observations. If a star is an unresolved double, the Hipparcos proper motion is polluted by the effect of the orbital motion on the photocentre, while this effect is smoothed out in FK5. So in some cases, the uncertainty of the Hipparcos proper motion, considered as the proper motion of the barycentre of the system, is underestimated. But by no means does this imply a systematic kinematic effect.

\section{References}

ESA 1997, The Hipparcos and Tycho Catalogues, ESA Publ. SP-1200.

Kovalevsky, J., Lindegren, L., Perryman, M.A.C. et al., 1997, A\&A, 323, 620-633.

Ma, C. and Feissel, M., 1997, Technical Note 23, Observatoire de Paris, June 1997. 